\title{
Some Applications of the Non Propagation Theorem
}

\author{
Nassim Athmouni, Mondher Damak \\ Département de Mathématiques, Faculté des Sciences de Sfax, Université de Sfax, Sfax, Tunisia \\ Email: Mondher.Damak@fss.rnu.tn
}

Received September 23, 2011; revised October 26, 2011; accepted November 5, 2011

\begin{abstract}
We use $C^{*}$-algebras to determine non-propagation estimates for a certain class of generalized Schrödinger operators acting on $L^{2}(X)$, where $X$ is a locally compact group. In particular, the Schrödinger operators on trees are included.
\end{abstract}

Keywords: $C^{*}$-Algebra; Schrödinger Operator; Propagation Properties; Tree

\section{Introduction}

It is well known that the algebraic approach leads to very interesting results on the spectral analysis of self-adjoint operators, as relevant results we quote: the essential spectrum and the Mourre estimate. A good expositions of this formalism and some applications may be found in [1-7]. We point out only that the main idea consists in showing that these operators are affiliated to suitable $C^{*}$-algebras which reflect their common properties well. A study of the quotient of these $C^{*}$-algebras by the ideal of compact operators leads to formula for the essential spectrum of these operators expressed as a union of spectra of some asymptotic operators. The quotient of the same $C^{*}$-algebras by other ideals give localization results of these operators which can be interpreted as non propagation properties of their unitary groups. The last result is discovered and developed in [8-11].

Let $H$ be a self-adjoint operator in Hilbert space $\mathcal{H}$ and $\chi$ a nontrivial multiplication operator (for example the characteristic function of a set having a strictly positive measure). If $\kappa$ is a continuous function with support intersecting the spectrum of $H$, the operator $\chi \kappa(H)$ has no reason to be small in general. The unique a priori bound would be

$$
\|\chi \kappa(H)\| \leq\|\chi\|_{\infty} \sup _{\lambda \in \sigma(H)}|\kappa(\lambda)|,
$$

where $\sigma(H)$ denotes the spectrum of $H$. We are going to correlate $\chi$ to $\kappa$ in such a way to make the norm small without asking any of the two factors on the right hand side of the preceding inequality to be small.

In order to understand the problem better we recall the following example (see [9] for more detail). In $\mathcal{H}=L^{2}(\mathbb{R})$ we consider the Schrödinger operator $H=H_{0}+V$, where $H_{0}$ is the positive Laplace operator and $V$ is the operator of multiplication with a bounded, uniformly continuous function having a limit at plus infinity: $V(x) \rightarrow c$ when $x \rightarrow+\infty$. Then we have that $[c, \infty)$ is included in the essential spectrum of $H$. The behavior of $V$ to the left may introduce a spectrum and even an essential spectrum below $c$. Now, let $\kappa: \mathbb{R} \rightarrow \mathbb{R}$ be continuous, with a compact support situated below the value $c$ and $\left\{\chi_{a}, a \in \mathbb{R}\right\}$ the family of all operators of multiplication with characteristic functions of intervals of the form $[a, \infty)$. Then we have that for any $\varepsilon>0$ there exists $a \in \mathbb{R}$, positive and sufficiently large, such that $\left\|\chi_{a} \kappa(H)\right\| \leq \varepsilon$. As a consequence we get the following non propagation result: $\left\|\chi_{a} \mathrm{e}^{-i t H} \kappa(H) f\right\| \leq \varepsilon\|f\|$, uniformly in $t \in \mathbb{R}$ and $f \in \mathcal{H}$. So, at energies below $c$, even when propagation towards infinity is possible, it does not take place to the right.

The purpose of this paper is to determine the localization properties for a class of generalized Schrödinger operators of the form $H=h(P)+V$ acting in the Hilbert space $\mathcal{H}=L^{2}(X)$ with $X$ is an abelian locally compact group. Here $P$ is the momentum operator, $h: X \rightarrow \mathbb{R}$ is a continuous function verifying some suitable hypothesis and $V: X \rightarrow \mathbb{R}$ is a continuous function having a certain type of anisotropic behavior at infinity. More precisely we shall show that: If $\kappa$ is a continuous real function with suitable support and if $\varepsilon>0$, then there exists a family of multiplication operators $\left\{\chi_{W}\right\}_{W \in W}$ where $W$ is a suitable family of subsets of $X$, such that $\left\|\chi_{W} \kappa(H)\right\| \leq \varepsilon$ for some $W \in W$. This can be reformulated in terms of the evolution group $\left\{\mathrm{e}^{-i t H}\right\}_{t \in \mathbb{R}}$ : at energies belonging to $\operatorname{supp}(\kappa)$, the system governed by $H$ stays "out $\operatorname{supp}\left(\chi_{W}\right)$ " uniformly in time. Practically, $\operatorname{supp}(\kappa)$ must not intersect the spectrum of a certain subhamiltonian associated to some ideal of the $C^{*}$ algebra in which the Hamiltonian $H$ is affiliated.

Let us describe the content of this paper. In Section 2 we introduce the framework and recall some definitions and results on crossed product $C^{*}$-algebra that are useful 
in the spectral theory of self-adjoint operators. Section 3 is devoted to the proof of non propagation result (Theorem 3.1) on the locally compact group, generalising the results of [8] where $X=\mathbb{R}^{n}$. As applications we study the multi-crystalline system and the Schrödinger operators with potential having a cartesian anisotropy in $l^{2}\left(\mathbb{Z}^{2}\right)$. Section 4 is mainly dedicated to study the non propagation theorem on trees (Theorem 4.1). Finally, we mention that certain generalizations can be made in the graph case (see [12]).

\section{Framework}

\subsection{The $\mathfrak{K}$-Essential Spectra}

Let $H$ be a self-adjoint operator in Hilbert space $\mathcal{H}$, by using the spectral theorem we can to associate an operator $\eta(H)$ to a large class of functions $\eta: \mathbb{R} \rightarrow \mathbb{C}$. We denote by $C_{0}(\mathbb{R})$ the set of all continuous functions $\eta: \mathbb{R} \rightarrow \mathbb{C}$ that vanish at infinity (i.e. satisfying

$\left.\lim _{x \rightarrow \pm \infty} \eta(x)=0\right)$. Some parts of the spectrum of $H$ can easily be expressed in terms of these functions: 1) a number $\lambda \in \mathbb{R}$ belongs to the spectrum $\sigma(H)$ of $H$ if $\eta(H)) \neq 0$ whenever $\eta \in C_{0}(\mathbb{R})$ and $\eta(\lambda) \neq 0$;2) $\lambda$ belongs to the essential spectrum $\sigma_{\text {ess }}(H)$ of $H$ if $\eta(H)$ is a non-compact operator whenever $\eta \in C_{0}(\mathbb{R})$ and $\eta(\lambda) \neq 0$.

Given $C^{*}$-subalgebra $\mathfrak{C}$ of bounded operators in $\mathcal{H}$. One says that $H$ is affiliated to $\mathfrak{C}$ if $(H-z)^{-1} \in \mathfrak{C}$ for some complex number $z$ outside the spectrum of $H$. If this is the case then $\varphi(H) \in \mathfrak{C}$ for each continuous function on $\mathbb{R}$ which tends to zero at infinity.

We recall that an observable affiliated to a $C^{*}$-algebra $\mathfrak{C}$ is a *-homomorphism from the $C^{*}$-algebra $C_{0}(\mathbb{R})$ to $\mathfrak{C}$ (i.e. a linear mapping $\Phi: C_{0}(\mathbb{R}) \rightarrow \mathfrak{C}$ satisfying $\Phi(\xi \eta)=\Phi(\xi) \Phi(\eta)$ and $\Phi(\eta)^{*}=\Phi(\bar{\eta})$ if $\xi$, $\left.\eta \in C_{0}(\mathbb{R})\right)$.

The spectrum $\sigma(\Phi)$ of the observable $\Phi$ is defined as the set of real numbers $\lambda$ such that $\Phi(\eta) \neq 0$ whenever $\eta(\lambda) \neq 0 . \quad \sigma(\Phi)$ is closed subset of $\mathbb{R}$.

Now let $\mathfrak{K}$ be a (closed, self-adjoint, bilateral) ideal in $\mathfrak{C}$. We denote by $\hat{\mathfrak{C}} \equiv \mathfrak{C} / \mathfrak{K}$ the associated quotient $C^{*}$-algebra and by $\Pi$ the canonical ${ }^{*}$-homomorphism of $\mathfrak{C}$ onto $\hat{\mathfrak{C}}$. If $\Phi$ is an observable affiliated to $\mathfrak{C}$, then clearly $П о \Phi$ determines an observable affiliated to $\hat{\mathfrak{C}}$.

Definition 2.1. The spectrum $\sigma(\Pi o \Phi)$ of the $o b-$ servable ПoФ (relative to $\hat{\mathfrak{C}}$ ) is called the $\mathfrak{K}$-essential spectrum of $\Phi$ and will be denoted by $\sigma_{\mathfrak{K}}(\Phi)$ : $\sigma_{\mathfrak{K}}(\Phi) \equiv \sigma(\Pi \circ \Phi)$. Equivalently, a real number $\lambda$ belongs to $\sigma_{\mathfrak{K}}(\Phi)$ if and only if $\Phi(\eta) \notin \mathfrak{K}$ whenever $\eta \in C_{0}(\mathbb{R})$ is such that $\eta(\lambda) \neq 0$.

Let $\mathfrak{C}$ be a $C^{*}$-subalgebra of the $C^{*}$-algebra $B(\mathcal{H})$ of bounded operators in $\mathcal{H}$ and $\Phi^{H}$ is the observable determined by a self-adjoint operator $H$ affiliated to $\mathfrak{C}$ (so $\Phi^{H}(\eta)=\eta(H)$ ). We assume that the ideal $K(\mathcal{H})$ of all compact operators in $\mathcal{H}$ is contained in $\mathfrak{C}$. The notion of the spectrum has obvious meaning and it is easy to show that the spectrum of $\sigma_{K(\mathcal{H})}\left(\Phi^{H}\right)$ is just the essential spectrum $\sigma_{e s s}(H)$ of the self-adjoint operator $H$.

It is obvious that, if $\mathfrak{K}_{1}$ and $\mathfrak{K}_{2}$ are two ideals in $\mathfrak{C}$ satisfying, $\mathfrak{K}_{1} \subset \mathfrak{K}_{2}$, then

$\sigma_{\mathfrak{K}_{2}}(\Phi) \subset \sigma_{\mathfrak{K}_{1}}(\Phi) \subset \sigma(\Phi)$. Furthermore, if $H$ is a self-adjoint operator affiliated to a $C^{*}$-subalgebra $\mathfrak{C}$ of $B(\mathcal{H})$ and if $\mathfrak{K}$ is an ideal in $\mathfrak{C}$ with $K(\mathcal{H}) \subset \mathfrak{K}$, then $\sigma_{\mathfrak{K}}\left(\Phi^{H}\right) \subset \sigma_{\text {ess }}(H)$.

The following Lemma was established Lemma 1 in [8], and will be used in subsection 3.1.

Lemma 2.2. Let $\mathfrak{K}$ be an ideal in a $C^{*}$-algebra $\mathfrak{C}$ and $\Phi$ an observable affiliated to $\mathfrak{C}$. If $\eta \in C_{0}(\mathbb{R})$ is such that $\eta(\mu)=0$ for all $\mu \in \sigma_{\mathfrak{K}}(\Phi)$, then $\Phi(\eta) \in \mathfrak{K}$.

\subsection{Locally Compact Space and $C^{*}$-Algebra}

Let $Y$ be locally compact, Hausdorff space, and $C_{b}(Y)$ the abelian $C^{*}$-algebra of all bounded, continuous complex functions defined on $Y$. If $G$ is a closed subset of $Y$, we set $C^{G}(Y)=\left\{\varphi \in C_{b}(Y) \mid \varphi(y)=0, \forall y \in G\right\}$. Certain $C^{*}$-subalgebras of $C_{b}(Y)$ will be useful, more precisely particular the algebra $C_{b}^{u}(Y)$ and $C_{0}(Y)$ consisting respectively of all bounded, uniformly continuous functions and of all continuous functions vanishing at infinity. In fact $C_{0}(Y)$ is an ideal of $C_{b}(Y)$.

In this paper we set $X$, a locally compact space and assume that $X$ acts on $Y$ by homeomorphisms: therefore if $\alpha_{x}$ denotes the homeomorphism in $Y$ associated to the element $x \in X$, we have $\alpha_{x} o \alpha_{x^{\prime}}=\alpha_{x+x^{\prime}}$. The mapping $X \times Y \rightarrow Y \quad(x, y) \mapsto \alpha_{x}(y)$ is assumed continuous. Then $\alpha$ induces a representation of the group $X$ by "-automorphism of $C_{b}(Y)$ as well as of various $C^{*}$ subalgebras of $C_{b}(Y):$ for $\varphi \in C_{b}(Y)$ and $x \in X$, define $a_{x}(\varphi)$ by $\left[a_{x}(\varphi)\right](y)=\varphi\left(\alpha_{x}(y)\right)(y \in Y)$.

Given a unital $C^{*}$-subalgebra $\mathcal{A}$ of $C_{b}(X)$ containing $C_{0}(X)$. We denote its character space by $\mathcal{X}$ and we recall that $\mathcal{X}$ is compactification of $X$. For $x \in X$, the character $i(x)$ is given by the formula $[i(x)](\varphi)=\varphi(x)$, for each $\varphi \in \mathcal{A}$. We denote by $\mathcal{Z}$ the frontier of $X$ in $\mathcal{X}$. By using the Gel'fand transformation we can observe that $\mathcal{A}$ is isomorphism to the $C^{*}$-algebra $C(\mathcal{X})$ of continuous function on $\mathcal{X}$. Henceforth we will be using the notions $\mathcal{G}: C(\mathcal{X}) \rightarrow \mathcal{A}$ for the inverse of Gel'fand isomorphism. The $C^{*}$-subalgebra $C^{\mathcal{Z}}(X)$ (consisting of continuous functions on $\mathcal{X}$ that vanish on the frontier $\mathcal{Z}$ of $\mathcal{X}$ ) can be naturally identified with $C_{0}(X)$, more precisely $C_{0}(X)=\mathcal{G} C^{\mathcal{Z}}(X)$. There is one-to-one correspondence between (self-adjoint, closed) ideals $\mathcal{K}$ of $\mathcal{A}$ and 
closed subsets $G$ of $\mathcal{X}$, given by $\mathcal{K}=\mathcal{G} C^{G}(\mathcal{X})$. In particular each closed subset $F$ of frontier $\mathcal{Z}$ determines an ideal $\mathcal{K}^{F}$ in $\mathcal{A}$, viz. $\mathcal{K}^{F}=\mathcal{G} C^{F}(\mathcal{X})$. It is clear that such an in ideal contains $C_{0}(X)$.

Set now that $C^{*}$-algebra $\mathcal{A}$ considered above is contained in $C_{b}^{u}(X)$, and invariant under translation, i.e. such that $a_{x} \mathcal{A} \subset \mathcal{A}$ for all $x \in X$, with $\left[a_{x}(\varphi)\right](y)=\varphi(x+y)$; such algebra will be called an $\mathrm{X}$-algebra. Since $\mathcal{A} \subset C_{b}^{u}(X)$, the mapping $x \mapsto a_{x}(\varphi)$ is norm continuous for each $\varphi \in \mathcal{A}$. Furthermore the action of $X$ on itself (given as $\alpha_{x}(y)=x+y$ ) induces a continuous. representation $\rho$ of $X$ by homeomorphisms of the character space $\mathcal{X}$ : for $\tau \in \mathcal{X}$ the character $\rho_{x} \tau$ is defined as $\left[\rho_{x} \tau\right](\varphi)=\tau\left[a_{x}(\varphi)\right]$. For $y \in X$, set $\tau_{y}=i(y)$; then $\rho_{x} \tau_{y}=\tau_{x+y} \quad(x \in X)$.

We end this section with a result which will be useful in the examples presented further on. Let $\tau \in \mathcal{X}$ be character of $\mathcal{A}$. A neighborhood base of $\tau \in \mathcal{X}$ is given by the collection $\left\{\mathcal{V}_{\mathcal{F}, \varepsilon}\right\}$, where $\varepsilon$ varies over $(0, \infty)$ and $\mathcal{F}$ over all finite families $\left\{\varphi_{1}, \cdots, \varphi_{m}\right\}$ of elements of $\mathcal{A}$ where

$$
\mathcal{V}_{\mathcal{F}, \varepsilon}(\tau)=\left\{\tau^{\prime}|| \tau^{\prime}\left(\varphi_{i}\right)-\tau\left(\varphi_{i}\right) \mid<\varepsilon \text { for each } \varphi_{i} \in \mathcal{F}\right\}
$$

Lemma 2.3. [Lemma 2, in AMP] Let $\mathcal{A}$ be a unital $C^{*}$-subalgebra of $C_{b}(X)$. Let $F$ be a closed subset of $\mathcal{X}$ and $\mathcal{W}$ a neighborhood of $F$. Then there exist $\varepsilon>0$ and a finite family $\mathcal{F}=\left\{\varphi_{1}, \cdots, \varphi_{m}\right\}$ of elements of $\mathcal{A}$ such that $F \subset \cup_{\tau \in F} \mathcal{V}_{\mathcal{F}, \varepsilon}(\tau) \subset \mathcal{W}$.

\subsection{Crossed Product $C^{*}$-Algebra}

If $\mathfrak{A}, \mathfrak{B}$ are subspaces of an algebra $\mathfrak{C}$ then we denote by $\mathfrak{A} \cdot \mathfrak{B}$ the linear subspaces of $\mathfrak{C}$ generated by the element $A B$ with $A \in \mathfrak{A}, B \in \mathfrak{B}$; if $\mathfrak{C}$ is a $C^{*}$ algebra then the norm closure of subspace $\mathfrak{A} \cdot \mathfrak{B}$ is denoted by $\langle\mathfrak{A} \cdot \mathfrak{B}\rangle$ and called crossed product of $\mathfrak{A}$ and $\mathfrak{B}$.

We consider some $C^{*}$-algebras of the space $B(\mathcal{H})$ of all bounded operators in the Hilbert space $\mathcal{H}=L^{2}(X)$. If $\varphi: X \rightarrow \mathbb{C}$ is a bounded, measurable function, we denote by $\varphi(Q)$ the operator of multiplication by $\varphi$ in $\mathcal{H}$ and by $\varphi(P)$ the operator $\mathcal{F}^{*} \varphi(Q) \mathcal{F}$, where $\mathcal{F}$ is the Fourier transformation. A $C^{*}$-subalgebra $\mathcal{A}$ of $C_{b}^{u}(X)$ will be identified with the subalgebra of $B(\mathcal{H})$ consisting of all multiplication operators $\varphi(Q)$ with $\varphi \in \mathcal{A}$.

If $\mathcal{A}$ is a $C^{*}$-algebra of $C_{b}^{u}(X)$, we write $\mathfrak{C}^{A}$ for norm closure in $B(\mathcal{H})$ of the set of finite sums of the form $\varphi_{1}(Q) \psi_{1}(P)+\cdots+\varphi_{n}(Q) \psi_{n}(P)$ with $\varphi_{k} \in \mathcal{A}$ and $\psi_{k} \in C_{0}(X)$. We mention the fact that, if $\mathcal{A}=C_{0}(X)$ then $\mathfrak{C}_{\mathcal{A}}$ is the ideal of all compact operators in $L^{2}(X)$. If $\mathcal{A}$ is a X-algebra, then $\mathfrak{C}_{\mathcal{A}}$ is a $C^{*}$-algebra isomorphic to the crossed product $\mathcal{A} \rtimes X$ in terms $a_{x}$ of $X$ on $\mathcal{A}$.
We recall now a result from [3]:

Theorem 2.4. Let $\mathcal{A}$ the X-algebra of the first kind and let $h: X^{*} \rightarrow \mathbb{R}$ be a continuous function such that $|h(k)| \rightarrow \infty$ where $|k| \rightarrow \infty$ in $X^{*}$. Then the $C^{*}$-subalgebra of $\boldsymbol{B}(\mathcal{H})$ generated by the self-adjoint operator of the form $h(P+k)+V(Q)$, where $k \in X^{*}$ and $V: X \rightarrow \mathbb{R}$ belongs to $\mathcal{A}$, coincide with $\mathcal{A} \rtimes X$.

\section{Non Propagation Theorem in the Locally Compact Groups}

\subsection{Main Result}

Let $X$ be a locally compact group. Let $\mathcal{A}$ be a unital $\mathrm{X}$-algebra of the first kind, $\mathcal{X}$ its character space, $F$ a translation invariant, closed subset of $\mathcal{Z}=\mathcal{X} \backslash X$, and $C^{F}(\mathcal{X})$ the ideal in $C(\mathcal{X})$ determined by $F$. Let $\mathfrak{C}_{A}=\mathcal{A} \rtimes X$ be the crossed product $C^{*}$-subalgebra of $B(\mathcal{H})$. We consider $\mathcal{K}^{F} \equiv \mathcal{G} C^{F}(\mathcal{X})$, which is a translation invariant ideal in $\mathcal{A}$, so $\mathfrak{K}^{F} \equiv \mathfrak{C}_{\mathcal{K}^{F}}$ is an ideal in $\mathfrak{C}_{\mathcal{A}}$ that contains all the compact operators in $\mathcal{H}$.

One has need to work with families $W$ of subsets of $X$ such that their images through $i$ in $\mathcal{X}$ are close to $F$. $W$ will have the structure of filter base. If $W$ is a filter base in $X$, then the family $\{i(W) \mid W \in \boldsymbol{W}\}$ is a filter base in $\mathcal{X}$ and we say that $W$ is adjacent to $F$ if all cluster points in $\mathcal{X}$ of this family $\{i(W) \mid W \in W\}$ belong to $F$, i.e. if $\cap_{W \in F} \overline{i(W)} \subset F$, where the closures are taken in $\mathcal{X}$. The set of these cluster points is non-empty since $\mathcal{X}$ is a compact space. In the majority of situations considered further on, it is enough to take for $W$ the family $\left\{W=i^{-1}[\mathcal{W} \cap i(X)] \mid \mathcal{W} \in \mathfrak{W}\right\}$, where $\mathfrak{W}$ is a neighborhood base of $F$ in $\mathcal{X}$ (since $i(X)$ is dense in $\mathcal{X}$, each of these sets $W$ is non-void).

If $W$ is a filter base adjacent to $F$ and $\varphi \in C(\mathcal{X})$ is such that $\varphi_{\mid F}=0$, then given any $\varepsilon>0$, there is some $W \in W$ such that $|\varphi(\tau)|<\varepsilon$ for all $\tau \in i(W)$. In the sequel we shall denote by $\chi_{W}$ the characteristic function of $W$.

The main result of this paper is the following non propagation theorem.

Theorem 3.1. Let $\mathcal{A}$ and $F$ be as above and $\boldsymbol{W}$ a filter base in $X$ that is adjacent to $F$. Let $L$ be a self-adjoint operator in $\mathcal{H}$ affiliated to $\mathfrak{C}_{A}$. Let $\varepsilon>0$ and $\kappa \in C_{0}(X)$ with $\operatorname{supp}(\kappa) \cap \sigma_{\mathfrak{K}_{F}}(L)=\varnothing$. Then there is a $W \in W$ such that

$$
\left\|\chi_{W}(Q) \kappa(L)\right\| \leq \varepsilon .
$$

In particular, one has:

$$
\left\|\chi_{W}(Q) e^{-i t L} \kappa(L) f\right\| \leq \varepsilon\|f\|
$$

uniformly in $t \in \mathbb{R}$ and for each $f$ in $L^{2}(X)$.

Proof. Our proof is similar to that of Theorem 1, in 
[8].

1) We use the notation $\tau$ for characters in $\mathcal{X}$ and observe that

$$
\begin{aligned}
2 \mathcal{K}^{F} & =\mathcal{G}\left\{\varphi \in C(\chi), \varphi_{\mid F} \equiv 0\right\} \\
& =\{\varphi \in \mathcal{A}, \tau(\varphi)=0 \forall \tau \in F\} .
\end{aligned}
$$

So if $\varphi$ belongs to $\mathcal{K}^{F}$, then for each $\delta>0$ there exists $W \in W$ such that $|\tau(\varphi)| \leq \delta \quad \forall \tau \in i(W)$. Thus, if $\varphi \in \mathcal{K}^{F}$, we have $|\varphi(x)| \leq \delta$ for all $x \in W$.

2) By the hypothesis on the support of $\kappa$ we have $\kappa(L) \in \mathfrak{K}^{F}$ (see Lemma 2.2). So there is a finite number of functions $\varphi_{1}, \cdots, \varphi_{m} \in \mathcal{K}^{F}$ and $\varphi_{1}, \cdots, \varphi_{m} \in C_{0}\left(X^{*}\right)$ such that

$$
\left\|\kappa(L)-\sum_{i=1}^{m} \varphi_{i}(Q) \psi_{i}(P)\right\| \leq \varepsilon / 2 .
$$

We have:

$$
\begin{aligned}
2\left\|\chi_{W}(Q) \kappa(L)\right\| \leq & \sum_{i=1}^{m}\left\|\varphi_{i}\right\|_{L^{\infty}(W)}\left\|\psi_{i}\right\|_{L^{\infty}\left(X^{*}\right)} \\
& +\left\|\kappa(L)-\sum_{i=1}^{m} \varphi_{i}(Q) \psi_{i}(P)\right\| .
\end{aligned}
$$

The first term in the r. h. s. of the preceding inequality can be made less than $\varepsilon / 2$ by using the result of 1) with $\delta=\left[m \sup _{i=1, \cdots, m}\left\|\psi_{i}\right\|_{L^{\infty}(X)}\right]^{-1} \frac{\varepsilon}{2}$, so the proof is finished.

The second result in the Theorem 3.1 gives the precise meaning of the notion of non-propagation described in the Introduction. To be more specific, let us denote by $\operatorname{supp}(f ; L)$ the spectral support with respect to $L$ of the vector $f \in \mathcal{H}$, defined as follows in terms of the spectral measure $E_{L}$ of $L$ :

$$
\begin{aligned}
& \lambda \notin \operatorname{supp}(f ; L) \text { iff } \exists \varepsilon>0 \\
& \text { suchthat } E^{L}(\lambda-\varepsilon, \lambda+\varepsilon) f=0 .
\end{aligned}
$$

$\operatorname{supp}(f ; L)$ is the smallest closed set $J \subset \mathbb{R}$ such that $E_{L}(J) f=f$. Then it follows easily that, under the hypotheses of the Theorem 3.1, for each $\varepsilon>0$ and each closed subset $A$ of $\mathbb{R} \backslash \sigma_{\kappa_{F} F}\left(\Phi^{L}\right)$ there exists an element $W \in W$ such that

$$
\left\|\chi_{W}(Q) e^{-i t L} f\right\| \leq \varepsilon\|f\|,
$$

for all $t \in \mathbb{R}$ and for each $f$ in $L^{2}(X)$ with $\operatorname{supp}(f ; L) \subset A$.

In the situation just described, we take for $L$ a Schrödinger operator affiliated to $\mathfrak{C}_{\mathcal{A}}$. Then, if $f$ is a unit vector with $\operatorname{supp}(f ; L) \subset A$, one has

$\left\|\chi_{W}(Q) \mathrm{e}^{-i t L} f\right\| \leq \varepsilon$ for all $t \in \mathbb{R}$. The physical interpretation: the probability of finding $f$ localized in $W$ is less than $\varepsilon^{2}$ all times. Assume that $K$ is a compact subset of $X$ and the preceding vector $f$ belongs to the absolutely continuous subspace of $L$, then there is $t_{0} \in \mathbb{R}$ such that $\left\|\chi_{W}(Q) \mathrm{e}^{-i t L} f\right\| \leq \varepsilon$ for all $t>t_{0}$. Therfore $\left\|\chi_{K}(Q) \mathrm{e}^{-i t L} f\right\|^{2} \geq 1-2 \varepsilon^{2}$ for all $t>t_{0}$, which (for small $\varepsilon$ ) essentially means that $f$ describes a state that will propagate into complement $(K \cup W)^{\complement}$ of the set $K \cup W$. A similar conclusion is true even if $f$ belongs to the singularly continuous subspace of $L$, except that some averaging over time may be necessary, there are $0<t_{0}<t_{1}$ such that

$$
\left(t-t_{0}\right)^{-1} \int_{t_{0}}^{t}\left\|\chi_{K^{\complement} \cup W^{\complement}}(Q) e^{-i \tau L} f\right\|^{2} \mathrm{~d} \tau \geq 1-3 \varepsilon^{2}
$$

for all $t>t_{1}$.

\subsection{Applications}

\subsubsection{Non Propagation in Multicrystalline System}

Let $S$ be the unit sphere in $X=\mathbb{R}^{n}$. For $j=1, \cdots, m$ let $\Gamma_{j}$ a periodic lattice in $X$ and $\Sigma_{j}$ a non-empty open subset of $S$, with $\bar{\Sigma}_{j} \cap \bar{\Sigma}_{k}=\varnothing$ if $j \neq k$. We denote by $C_{j}(X)$ the $C^{*}$-algebra

$$
\begin{aligned}
C_{j}(X)= & \left\{\varphi \in C_{b}^{u}(X) ; \varphi(x+\gamma)=\varphi(x)\right. \\
& \left.\forall x \in X, \forall \gamma \in \Gamma_{j}\right\}
\end{aligned}
$$

and we define $\mathcal{A}$ as the set of bounded, uniformly continuous function $\varphi$ on $X$ such that for any $j \in\{1, \cdots, m\}$ there exists $\varphi_{j} \in C_{j}(X)$ such that

$$
\lim _{r \rightarrow \infty}\left|\varphi(r w)-\varphi_{j}(r w)\right|=0
$$

for all $w \in \Sigma_{j}$, uniformly in $w$ on each compact subset of $\Sigma_{j}$. The (uniquely determined) collection $\left\{\varphi_{j} \mid j=1, \cdots, m\right\}$ corresponding to $\varphi \in \mathcal{A}$ will be called the asymptotic function of $\varphi$. If $\Sigma$ is subset of $\Sigma_{j}$ and $R>0$, let $W_{j}^{R}(\Sigma)$ be the subset $\{r w \mid r>R, w \in \Sigma\}$ of $X$. We obtain the following result:

Proposition 3.2. Let $V \in \mathcal{A}$ be real and denote its asymptotic function by $\left\{V_{j}\right\}$. Set $L=h(P)+V$. Fix a number $j \in\{1, \cdots, m\}$ and choose $\kappa \in C_{0}(X)$ with $\operatorname{supp}(\kappa)$ disjoint from the spectrum of the periodic generalized Schrödinger operator $L_{j}=h(P)+V_{j}$. Then given a compact subset $K$ of $\Sigma_{j}$ and $\varepsilon>0$, there is $R \in(0, \infty)$ such that for each $f \in L^{2}(X)$ we have

$$
\sup _{t \in \mathbb{R}}\left\|\chi_{W_{j}^{R}(K)}(Q) \mathrm{e}^{-i t L} \kappa(L) f\right\| \leq \varepsilon\|f\| .
$$

For the proof of the Proposition we will need the following notations: $W_{j}^{R}\left(\Sigma_{j}\right)=\left\{r w \mid r>R>0, w \in \Sigma_{j}\right\}$, and $\mathbb{T}_{j}=X / \Gamma_{j}$ (The class of $z \in X$ in $\mathbb{T}_{j}$, denoted by $\zeta$, is given as $\left.\zeta=\left\{x \in X, \mid x=z+\gamma, \gamma \in \Gamma_{j}\right\}\right)$. We observe that $C_{j}(X)$ is isomorphic to $C\left(\mathbb{T}_{j}\right)$ and that the correspondence $\varphi \mapsto \varphi_{j}$ defines a *-homomorphism $\Psi_{j}$ from $\mathcal{A}$ to $C_{j}(X)$.

Proof. We consider the mapping: 


$$
\begin{gathered}
i_{j}: \mathbb{T}_{j} \rightarrow \mathcal{X} \\
\zeta \mapsto \tau_{\zeta}^{j}: \tau_{\zeta}^{j}(\varphi)=\varphi_{j}(z),
\end{gathered}
$$

where $\varphi_{j}$ is the $j$-th asymptotic function of $\varphi$ and $z$ any representative of the class $\zeta$. Set $\mathcal{T}_{j}=i_{j}\left(\mathbb{T}_{j}\right)$. By Lemma 4 in $A M P, \mathcal{T}_{j}$ is closed invariant under all translation subsets of $\mathcal{X} \backslash i(X)$. Now, we need the next result: The set $\sigma_{\mathfrak{K}^{\tau_{j}}}(L)$ coincides with the spectrum of the operator $L_{j}$. Indeed, let $\Pi_{j}: \mathfrak{C}_{\mathcal{A}} \rightarrow \mathfrak{C}_{\mathcal{A}} / \mathfrak{K}^{\mathcal{T}}$ the canonical *-morphism and denote by $\sigma_{\mathfrak{K}^{\mathcal{T}_{j}}}(L)$ the spectrum of the observable $\Pi_{j} o \Phi^{L}$ affiliated to $\mathfrak{C}_{\mathcal{A}} / \mathfrak{K}^{\mathcal{T}_{j}}$. It suffices to prove that $\mathfrak{C}_{\mathcal{A}} / \mathfrak{K}^{\mathcal{T}_{j}} \cong \mathfrak{C}_{C_{j}(X)}$ and the image of $\Pi_{j} o \Phi^{L}$ by this isomorphism is $\Phi_{j}^{L}$.

Set

$$
\Theta_{j}\left[\sum_{i=1}^{m} \varphi_{i}(P) \psi_{i}(Q)\right]=\sum_{i=1}^{m} \varphi_{i, j}(P) \psi_{i}(Q),
$$

where $\varphi_{i, j} \in C_{j}(X)$ is the $j^{\text {th }}$ asymptotic function of $\varphi_{i} . \Theta_{j}$ can be extended to the surjective morphism from $\mathfrak{C}_{\mathcal{A}}$ to $\mathfrak{C}_{C_{j}(X)}$, its kernel is $\mathfrak{K}^{\mathcal{T}_{j}}=C^{\mathcal{T}_{j}}(\mathcal{X}) \rtimes X$ (see Corollary 2.7. in [4]). Since $\mathcal{A}$ is a unital $X$-algebra of the first kind (see Lemma 3 in $A M P$ ), then by Theorem 2.4, we get

$$
\Theta_{j}\left((L-z)^{-1}\right)=\left(L_{j}-z\right)^{-1}
$$

By the preceding result we have $\operatorname{supp}(\kappa) \cap \sigma_{\mathfrak{K}^{\tau_{j}}}(L)=\varnothing$. Let $\mathfrak{W}$ be a neighbourhood base of $\mathcal{T}_{j}$ in $\mathcal{X}$. We consider the filter base defined by

$$
\boldsymbol{W}=\left\{W=i^{-1}[\mathcal{W} \cap i(X)] \mid \mathcal{W} \in \mathfrak{W}\right\} .
$$

Then by Theorem 3.1, there is $\varepsilon>0$ and $W \in W$ such that

$$
\left\|\chi_{W}(Q) \mathrm{e}^{-i t L} \kappa(L) f\right\| \leq \varepsilon\|f\|,
$$

uniformly in $t \in \mathbb{R}$ and for each $f$ in $L^{2}(X)$. To complete the proof we apply Lemma 5 in $A M P$.

\subsubsection{Potential with Cartesian Anisotropy}

Let $X=\mathbb{Z}^{2} \equiv \mathbb{Z}_{1} \times \mathbb{Z}_{2}$. For $j=1,2$ let us denote by $\mathcal{A}_{j}$ the $C^{*}$-algebra of all continuous functions $\varphi: \mathbb{Z}_{j} \rightarrow \mathbb{C}$ such that the limits $\lim _{n_{*} \pm \infty} \varphi\left(n_{j}\right)=c^{ \pm}$exists. Then $\mathcal{A}=\mathcal{A}_{1} \otimes \mathcal{A}_{2}$ is a unital $C^{*}$-subalgebra of $C_{b}^{u}\left(\mathbb{Z}^{2}\right)$ that is invariant under translations by elements of $X$.

Let $\overline{\mathbb{Z}}_{j}=\mathbb{Z}_{j} \cup\left\{-\infty_{j},+\infty{ }_{j}\right\}$ the two-point compactification of $\mathbb{Z}_{j}$. Then $\overline{\mathbb{Z}}_{j}$ is the spectrum of $\mathcal{A}_{j}$ and $\mathcal{X}=\overline{\mathbb{Z}}_{1} \times \overline{\mathbb{Z}}_{2}$ is the spectrum of $\mathcal{A}$. Now we consider the self-adjoint operator $L=-\Delta+V$ in $\mathcal{H}=l^{2}\left(\mathbb{Z}^{2}\right)$ for
$V \in \mathcal{A}$ real function. It is obvious that for $j, k \in\{1,2\}$ with $j \neq k$ the limits $V_{j}^{ \pm}\left(n_{j}\right)=\lim _{n_{k} \rightarrow \infty_{\infty}} V(n)$ exist uniformly in $n_{j} \in \mathbb{Z}_{j}$, and define elements of $\mathcal{A}_{j}$. Let $L^{+}=\left(-\Delta_{1}+V_{1}^{+}\right) \otimes I_{2}+I_{1} \otimes\left(-\Delta_{2}+V_{2}^{+}\right)$in the representation $l^{2}\left(\mathbb{Z}_{1}\right) \otimes l^{2}\left(\mathbb{Z}_{2}\right)$. Its spectrum equals $[c, 8+c]$ where $c=c_{1}^{+}+c_{2}^{+}, c_{1}^{+}=\lim _{n \rightarrow+\infty} V_{1}^{+}(n)$ and $c_{2}^{+}=\lim _{n \rightarrow+\infty} V_{2}^{+}(n)$.

Proposition 3.3. Let $\varepsilon>0$. Then for any $\kappa \in C(\mathbb{R})$ with supp $\kappa \cap[c, 8+c]=\varnothing$ there is $n_{\varepsilon} \in \mathbb{N}$ such that for all $n_{1}, n_{2}>n_{\varepsilon}$ and $f \in \mathcal{H}$,

$$
\left\|\chi\left(Q_{1}>n_{1}\right) \chi\left(Q_{2}>n_{2}\right) \mathrm{e}^{-i t L} \kappa(L) f\right\| \leq \varepsilon\|f\| .
$$

\subsection{Main Result in the Case of Trees}

Let $\Gamma$ be a $v$-fold tree of origin $e$ equipped with its canonical metric $d$, where $d(x, y)$ is the shortest path joining $x$ to $y$. We denote by $\mathrm{x} \sim \mathrm{y}$ when $\mathrm{x}$ and $\mathrm{y}$ are connected by any edge. For each $x \in \Gamma$, we denote by $x^{\prime}=$ $x^{(1)}$, the unique element $\mathrm{y} \sim \mathrm{x}$ such that $|y|=|x|-1$. For all $x \in \Gamma$,we define $|x|=d(e, x)$, Let $\mathcal{D}$ be the $C^{*}$-algebra generated by $\partial$. Where

$(\partial f) f(x)=\sum_{y^{\prime}=x} f(y)$, We denote by $\mathcal{C}(\hat{\Gamma})$ the $C^{*}$ algebra generated by $\mathcal{D}$ and $C(\hat{\Gamma})$ where $\hat{\Gamma}$ is the compacatification of $\Gamma$ and $C(\hat{\Gamma})$ is the $C^{*}$-algebra of the complex-valued continuous function on $\hat{\Gamma}$. It contains the compact operators on $\ell^{2}(\Gamma)$.

Let $L=\sum_{\alpha, \beta} a_{\alpha, \beta}(Q) \partial^{* \alpha} \partial^{\beta}+K$, where $a_{\alpha, \beta} \in C(\hat{\Gamma}), a_{\alpha, \beta}=0$ for almost all $(\alpha, \beta) \in \mathbb{N}^{2}$ and $K$ is a compact operator. Its clear that $L \in \mathcal{C}(\hat{\Gamma})$.

Theorem 3.4. Let $v>1$ and let

$L=\sum_{\alpha, \beta} a_{\alpha, \beta}(Q) \partial^{* \alpha} \partial^{\beta}+K$ where $K$ is a compact operator, $a_{\alpha, \beta} \in C(\hat{\Gamma}, \mathbb{R}), a_{\alpha, \beta}=0$ for almost all

$(\alpha, \beta) \in \mathbb{N}^{2}$, be a self adjoint operator. For all $n \in \mathbb{N}$ set

$$
B(n):=\{x \in \Gamma:|x| \geq n\} .
$$

Let $\kappa: \mathbb{R} \rightarrow \mathbb{R}$ be a continuous function with

$$
\operatorname{supp}(\kappa) \cap \bigcup_{\gamma \in \partial \Gamma} \sigma\left(\sum_{\alpha, \beta} a_{\alpha, \beta}(\gamma) \partial^{* \alpha} \partial^{\beta}\right)=\varnothing .
$$

Then for each $\varepsilon>0$ there exists $n_{\varepsilon} \in \mathbb{N}$ such that

$$
\left\|\chi_{B(n)} \kappa(L)\right\| \leq \varepsilon
$$

Proof. 1) Let

$$
\mathcal{K}^{\partial \Gamma}=\left\{\varphi \in C(\hat{\Gamma}) \mid \varphi_{\partial \Gamma \Gamma}=0\right\} .
$$

where $\partial \Gamma=\hat{\Gamma} / \Gamma$. So if $\varphi$ belongs to $\mathcal{K}^{\partial \Gamma}$ then for each $\delta>0$ there exists $n_{\varepsilon}$ such that $|\tau(\varphi)| \leq \delta \forall n \geq n_{\varepsilon}$.

2) Using Theorem 5.9 in [13] we can deduce that there is a unique morphism $\Phi: \mathcal{C}(\hat{\Gamma}) \rightarrow \mathcal{D} \otimes C(\partial \Gamma)$ such that $\Phi(D)=D \otimes 1$ for all $D \in \mathcal{D}$ and $\Phi(\varphi(Q))=1 \otimes\left(\varphi_{\partial \supset \Gamma}\right)$. Now, its clear that $\Phi$ is surjec- 
tive with kernel equal to $\mathfrak{K}^{\partial \Gamma}$, where $\mathfrak{K}^{\partial \Gamma}=\left\langle\mathcal{K}^{\partial \Gamma} \mathcal{D}\right\rangle$. Then

$$
\Phi(L)=\sum_{\alpha, \beta} \partial^{* \alpha} \partial^{\beta} \otimes\left(a_{\alpha, \beta}\right)_{\mid \sigma \Gamma}
$$

and as consequence we obtain

$$
\sigma_{e s s}(L)=\bigcup_{\gamma \in \partial \Gamma} \sigma\left(\sum_{\alpha, \beta} a_{\alpha, \beta}(\gamma) \partial^{* \alpha} \partial^{\beta}\right) .
$$

Now using the hypothesis on the support of $\kappa$ we have $\kappa(L) \in \mathfrak{K}^{\partial \Gamma}$ (see Lemma 2.2). So there is a finite number of functions $\phi_{1}, \cdots, \phi_{m} \in \mathcal{K}^{\partial \Gamma}$ such that

$$
\left\|\kappa(L)-\sum_{i=1}^{m} \varphi_{i}(Q) \partial^{* \alpha_{i}} \partial^{\beta_{i}}\right\| \leq \varepsilon / 2 .
$$

We have

$$
\begin{aligned}
2\left\|\chi_{B(n)}(Q) \kappa(L)\right\| \leq & \sum_{i=1}^{m}\left\|\varphi_{i}\right\|_{l^{\infty}(B(n))}\left\|\partial^{* \alpha_{i}} \partial^{\beta_{i}}\right\| \\
& +\left\|\kappa(L)-\sum_{i=1}^{m} \varphi_{i}(Q) \partial^{* \alpha_{i}} \partial^{\beta_{i}}\right\| .
\end{aligned}
$$

The first term in the r. h. s. of the preceding inequality can be made less than $\varepsilon / 2$ by using the result of 1) with $\delta=\left[m \sup _{i=1, \cdots, m} \alpha_{i} \beta_{i} v\right]^{-1} \frac{\varepsilon}{2}$, so the proof is finished.

Example 3.5. We can consider the Schrödinger operator $L=\Delta+V(Q)$ with potential $V$ in $C(\hat{\Gamma})$. Here $\Delta$ is the bounded operator defined on $\ell^{2}(\Gamma)$ by

$$
(\Delta f)(x)=\sum_{x \sim y}(f(y)-f(x)) .
$$

Then we have that $L \in \mathcal{C}(\hat{\Gamma})$ and (see [13]):

$$
\begin{aligned}
\sigma_{e s s}(\Delta+V(Q)) & =\sigma(\Delta)+V(\partial \Gamma) \\
& =[-v-2 \sqrt{v},-v+2 \sqrt{v}]+V(\partial \Gamma) .
\end{aligned}
$$

Then we obtain the conclusion of the theorem for each function $\kappa$ with support not intersect the set $[-v-2 \sqrt{v},-v+2 \sqrt{v}]+V(\partial \Gamma)$.

Corollary 3.6. Let $L$ and $\Gamma$ be as in Theorem 3.4. Then, for any $\varepsilon>0$, there exists $n_{\varepsilon} \in \mathbb{N}$ such that

$$
\left\|\chi_{B(n)} e^{-i t L} f\right\| \leq \varepsilon\|f\|
$$

for all $n \geq n_{\varepsilon}, t \in \mathbb{R}$ and all $f \in \ell^{2}(\Gamma)$ satisfying

$$
\operatorname{supp}(f ; L) \cap \bigcup_{\gamma \in \partial \Gamma} \sigma\left(\sum_{\alpha, \beta} a_{\alpha, \beta}(\gamma) \partial^{* \alpha} \partial^{\beta}\right)=\varnothing .
$$

\section{REFERENCES}

[1] W. Amrein, A. Boutet de Monvel and V. Georgescu, " $C_{0}$-Groups, Commutator Methods and Spectral Theory of $N$-Body Hamiltonians," In: H. Bass, J. Oesterlé, Y. Tschinkel and A. Weinstein, Eds., Progress in Mathematics, Vol. 135, Birkhäuser, Basel, 1996.

[2] M. Damak, "On the Spectral Theory of Depersive NBody Hamiltonians," Journal of Mathematical Physics, Vol. 40, No. 1, 1999, pp. 35-48. doi:10.1063/1.532759

[3] M. Damak and V. Georgescu, " $C$-Cross Products and a Generalized Mechanical N-Body Problem," Electronic Journal of Differential Equations Conference, Vol. 4, 2000, pp. 51-69.

[4] M. Măntoiu, "On the Class of Anisotropic Schrödinger Operators," Reviews in Mathematical Physics, to be submitted. http://www.ma.utexas.edu/mp-arc/

[5] M. Măntoiu, " $C^{*}$-Algebras, Dynamical Systems at Infinity and the Essential Spectrum of Generalized Schrödinger Operators," Journal fur die Reine und Angewandte Mathematik, Vol. 550, 2002, pp. 211-229.

[6] V. Georgescu and A. Iftimovici, "Crossed Products of $C^{*}$-Algebras and Spectral Analysis of Quantum Hamiltonians," Communications in Mathematical Physics, Vol. 228, No. 3, 2002, pp. 519-560. doi: $10.1007 / \mathrm{s} 002200200669$

[7] M. Damak and V. Georgescu, "Self-Adjoint Operators Affiliated to $C^{*}$-Algebras Generalized Mechanical," $R e$ views in Mathematical Physics, Vol. 16, 2004, pp. $257-$ 280. doi:10.1142/S0129055X04001984

[8] W. Amrein, M. Măntoiu and R. Purice, "Propagation Properties for Schrödinger Operators Affiliated with Certain $C^{*}$-Algebras," Annales Henri Poincaré, Vol. 3, No. 6, 2002, pp. 1215-1232.

[9] M. Măntoiu, " $C^{*}$-Algebras, Dynamical Systems, Spectral Analysis, Operator Algebras and Mathematical Physics," Constanta, Bucharest, 2003, pp. 299-314.

[10] M. Damak, M. Măntoiu and R. T. de Aldecoa, "Toeplitz Algebras and Spectral Results for the One Dimensional Hesenberg Model," Journal of Mathematical Physics, Vol. 47, 2006.

[11] M. Mantoiu, R. Purice and S. Richard, "Spectral and Propagation Results for Magnetic Schrödinger Operators; a $C^{*}$-Algebraic Framework," Journal of Functional Analysis, Vol. 250, No. 1, 2007, pp. 42-67. doi:10.1016/j.jfa.2007.05.020

[12] V. Georgescu and S. Golenia, "Isometries, Fock Spaces, and Spectral Analysis of Shrödinger Operators on Trees," Journal of Functional Analysis, Vol. 227, No. 2, 2005, pp. 389-429.

[13] S. Golenia, " $C^{*}$-Algebra of Anistropic Schrödinger Operators on Trees," Annales Henri Poincaré, Vol. 5, No. 6, 2004, pp. 1097-1115. 